\title{
Energy-Aware Routing Optimization in Dynamic GMPLS Controlled Optical Networks
}

Wang, Jiayuan; Ricciardi, Sergio; Fagertun, Anna Manolova; Ruepp, Sarah Renée; Careglio, Davide; Dittmann, Lars

\section{Published in:}

Proceedings of International Conference on Transparent Optical Networks (ICTON)

Publication date:

2012

Link back to DTU Orbit

Citation (APA):

Wang, J., Ricciardi, S., Fagertun, A. M., Ruepp, S. R., Careglio, D., \& Dittmann, L. (2012). Energy-Aware Routing Optimization in Dynamic GMPLS Controlled Optical Networks. In Proceedings of International Conference on Transparent Optical Networks (ICTON)

\section{General rights}

Copyright and moral rights for the publications made accessible in the public portal are retained by the authors and/or other copyright owners and it is a condition of accessing publications that users recognise and abide by the legal requirements associated with these rights.

- Users may download and print one copy of any publication from the public portal for the purpose of private study or research.

- You may not further distribute the material or use it for any profit-making activity or commercial gain

- You may freely distribute the URL identifying the publication in the public portal 


\title{
Energy-Aware Routing Optimization in Dynamic GMPLS Controlled Optical Networks
}

\author{
Jiayuan Wang, Sergio Ricciardi*, Anna Manolova Fagertun, Sarah Ruepp, Davide Careglio*, \\ and Lars Dittmann \\ Technical University of Denmark, Ørsteds Pl., 2800 Kgs. Lyngby, Denmark \\ * Technical University of Catalonia, C. Jordi Girona 1-3, 08034, Barcelona, Spain \\ Tel: (0045) 45257376,e-mail: jwan@fotonik.dtu.dk
}

\begin{abstract}
In this paper, routing optimizations based on energy sources are proposed in dynamic GMPLS controlled optical networks. The influences of re-routing and load balancing factors on the algorithm are evaluated, with a focus on different re-routing thresholds. Results from dynamic network simulations show that re-routing strategies can lower $\mathrm{CO}_{2}$ emissions compared to the basic energy source routing scheme, and a lower re-routing threshold achieves more savings. The increased blocking probability brought by using re-routing schemes can be compensated by applying load balancing criteria. A trade-off between blocking probability and obtained $\mathrm{CO}_{2}$ savings is studied.
\end{abstract}

Keywords: GMPLS, optical networks, energy-efficient, re-routing.

\section{INTRODUCTION}

With the rapid growth of the network bandwidth demand in the past years, concerns have arisen due to the accompanied growing energy consumption. The network components (routers, transponders, ROADMs, amplifiers, etc.) are becoming significant contributors to the greenhouse gas emissions. As an example, a $1 \mathrm{~kW}$ router powered by a coal power plant can contribute to 8.6 tons of $\mathrm{CO}_{2}$ emissions per year [1]. Thus, if the network traffic can be monitored to go through green routes (routes which use green energy sources such as wind or solar), the $\mathrm{CO}_{2}$ emissions can be greatly reduced. The recent development in Smart Grid technology has made it possible to select the type of energy sources (solar, wind, fuel, coal, etc.) used for network components, and some research has been done to employ green energy sources to reduce the network energy consumption, as in [2][3][4]. Ideas regarding re-routing strategies are also mentioned in [5], where the author focuses on turning off links in the network. The results are given in a static environment. Similar work done in [6] also aims at turning off unused links. However, the majority of the work done in the optical networking field is for a static network environment, with time-consuming optimization methods, such as linear programming. In this paper, a pure energy source aware (ESA) routing algorithm (routing decision based on the path with most green energy sources) is implemented to solve such issues in Generalized Multi-protocol Label Switching (GMPLS) [7] controlled dynamic optical core networks. Open Shortest Path First - Traffic Engineering (OSPF-TE) [8] is used as routing protocol, and Resource Reservation Protocol - Traffic Engineering (RSVP-TE) [9] is used as signalling protocol. In the considered environment, it is difficult to use the traditional optimization methods for improving the sub-optimal results, due to the unpredictability of the traffic pattern, the dynamically changing energy sources and the need for a fast convergence routing protocol. In this paper, besides presenting the results of ESA routing algorithm, with randomly changing energy sources (green and dirty), several optimization criteria are exploited under different energy source changing intervals, such as applying re-routing policies when a energy source has changed during the connection duration and the employment of load balancing factors in helping with the connection blocking rate. To the authors' best knowledge, this paper is one of the first works addressing these issues in a dynamic environment with re-optimization and load-balancing criteria.

The remainder of the paper is organized as follows. Section 2 presents the energy model used for estimating $\mathrm{CO}_{2}$ emissions; implementation environment is described in Section 3; simulation results are illustrated in Section 4; and conclusions are given in Section 5.

\section{ENERGY MODEL}

In order to evaluate the energy savings of the ESA algorithm, a simple energy model is employed to provide an estimation of the greenhouse gases emissions (green degree) of each connection. The optical network is assumed to be composed of network nodes (optical cross-connects, OXCs) and links, with optical amplifiers (OAs) to amplify optical signals every 80 kilometres. Green energy sources are assumed to emit zero $\mathrm{CO}_{2}$, while oil energy sources are assumed to emit 880 grams $/ \mathrm{kWh}$ and coal sources are assumed to emit $980 \mathrm{grams} / \mathrm{kWh}$. The energy consumption in a node is assumed to comprise of two components: a fixed one, due to the consumption of keeping device powered on and is independent of the traffic through the node, and a dynamic one, dependent on the type of the energy source and the amount of traffic through the node. The OA's energy consumption is assumed to be traffic independent, since the OA (like erbium-doped fibre amplifier (EDFA)) amplifies the entire C-band. 
Thus, the green degree of a light path $\mathrm{p}$ is given by (excluding the fixed part of consumption in a node):

$$
E_{p}=\left(\sum_{n \in p} e_{n} \cdot b_{p} \cdot t_{n}+\sum_{l \in p} \frac{e_{l}}{w_{l}} \cdot \frac{l_{l}}{\Lambda} \cdot t_{l}\right) \cdot d_{p},
$$

where $e_{n}$ is the energy consumption per Gbps in node $n, b_{p}$ is the bandwidth of the path in Gbps, $d_{p}$ is the duration of the connection, $t_{n}$ is the energy type of node $n\left(\mathrm{CO}_{2} / \mathrm{kWh}\right), e_{l}$ is the energy consumption of an OA on link $l, \omega_{l}$ is the wavelengths used on the link $l$ (used to amortize the OA over the set of active wavelengths), $l_{l}$ is the length of the link, $\Lambda$ is the maximum allowed link length without need of amplifying, and $t_{l}$ is the energy type of link $l\left(\mathrm{CO}_{2} / \mathrm{kWh}\right)$.

\section{SOFTWARE IMPLEMENTATION}

The proposed network environment is implemented in the event driven simulator - OPNET [10]. The network topology used in the simulations is the COST266 [11], with 37 nodes and 55 bidirectional links. Each link is assigned sixty-four wavelengths. The details of the simulation setup can be referenced in [11], with additional optical amplifiers placed every $80 \mathrm{~km}$ on each link. Label Switched Path (LSP) connection requests are generated as a Poisson process, with exponentially distributed connection duration. The mean value for the used connection duration is 6 hours, with mean inter-arrival time between connection requests of 30 minutes, which gives an offered load of 12 Erlangs per node. In the simulation model, first fit algorithm is applied for the wavelength assignment.

At fixed time intervals, a new update of the energy source information (TE-LSA [8]) is originated and flooded between neighbours using OSPF-TE extension. This fixed time interval is referred to as EnergyChangeInterval in this paper. Upon flooding the TE-LSA over the whole network, the new energy source information is updated in each LSA database, and thus, used for routing calculation.

Three performance metrics have been evaluated: Average $\mathrm{CO}_{2}$ emissions per day (Figure 1), average hop count number per connection (Figure 2) and average connection blocking probability (Figure 3). Besides ESA algorithm, the shortest path (SP) algorithm and pure load balancing algorithm (always choosing the least loaded links) are also applied as comparisons. Among them, four optimizations are applied on the ESA algorithm, adding re-routing and load balancing factors. Different re-routing thresholds, namely 30\%, 50\% and $70 \%$ are applied, indicating the minimum percentage of potential savings on the new route, if re-routing is performed. It is to say, when a re-routing is attempted, a potential new route is calculated first. If the emission on the new path is, e.g. $45 \%$, less than the old path, the re-routing decision will be made, in the case of $30 \%$ threshold. The cost function used for routing decision can be revised to take into account both energy sources and loads on the path by weighing the contribution of the cost due to energy sources and the cost due to load on the path according to: cost $=a^{*}$ cost_from_energy $+(1-a) *$ cost_from_load. By giving different values to $a$, we can achieve different optimization criteria. The ESA optimization case indicated as ESA + reroute $30 \%+$ load balancing $50 \%$ indicates that a $30 \%$ threshold is used for rerouting and $a$ is 0.5 (i.e. $50 \%$ of the routing cost is contributed to load balancing).

Five different update intervals (EnergyChangeInterval) are evaluated (1, 3, 6, 12, 24 hours), during 30 days of simulation runtime. Results are generated with $95 \%$ confidence intervals from 20 prime number seeds.

\section{RESULTS AND DISCUSSIONS}

Figure 1 shows the green degree resulting from different algorithms. As shown, $\mathrm{CO}_{2}$ emissions of SP algorithm remain almost unchanged with increasing EnergyChangeInterval, because energy source information has no effect on the routing decision made by using SP algorithm (i.e. SP is energy-unaware). ESA algorithm and its four optimizations only start to be greener than SP algorithm when the EnergyChangeInterval is less frequent. As the average connection duration is 6 hours, many energy source changes during the connection duration will eliminate the advantages brought by the initial routing decision based on the current green degree of the path. One natural optimization choice is to re-route the connection after the energy source on the path has changed. As expected, re-routing based on lower threshold results in higher savings. The savings by re-routing become less significant with increased EnergyChangeInterval, since less re-routing is requested when the energy source information is updated less frequent. The situation is the opposite for the pure load balancing algorithm. In order to select the less loaded paths, pure load balancing algorithm tend to pick up longer paths, which results in higher energy consumption. As the EnergyChangeInterval is also the update interval for updating the routing tables, the $\mathrm{CO}_{2}$ emissions become even higher when the routing updates are less frequent, as a result of the latency between the real network load information and the updated information. Thus, the hop count increases with the longer update interval, as shown in Figure 2. The $\mathrm{CO}_{2}$ emissions also increase correspondingly, as a result of longer paths. 


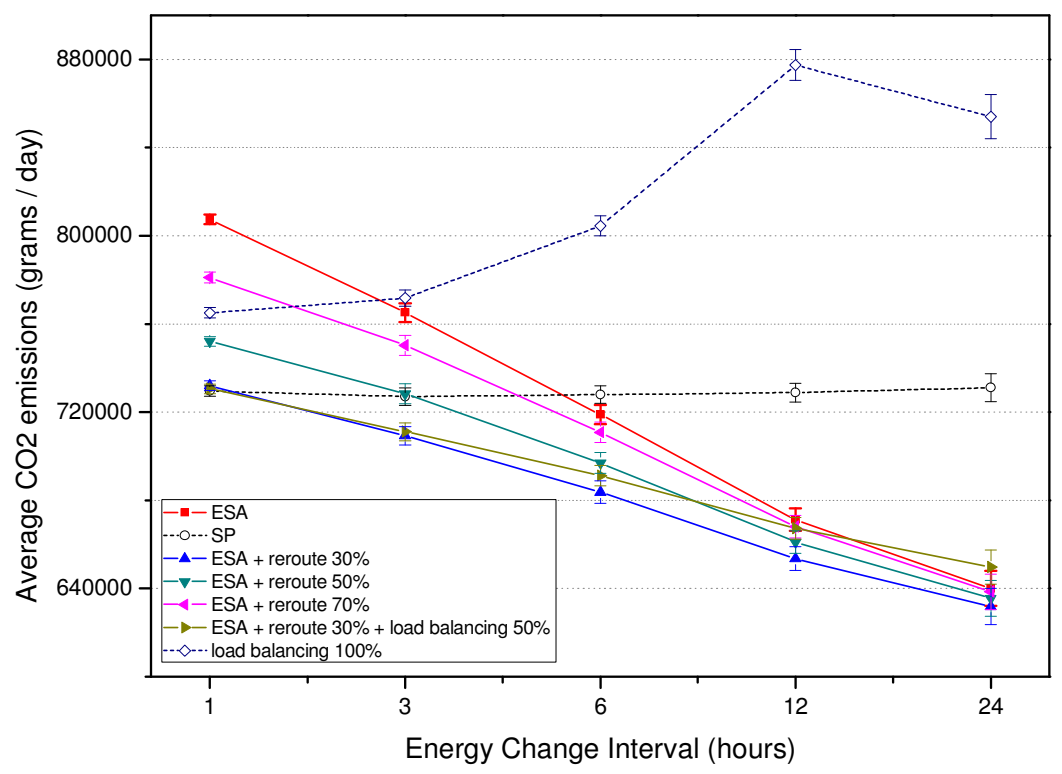

Figure 1. Average $\mathrm{CO}_{2}$ emissions per day.

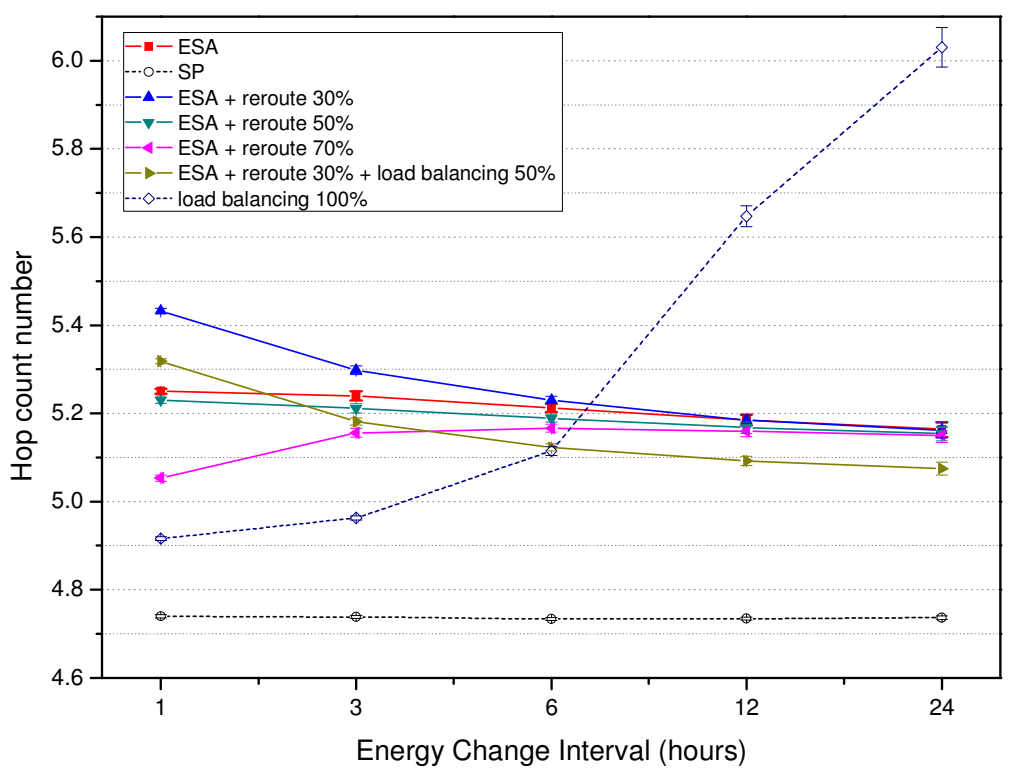

Figure 2. Average hop count number per connection.

Compared to the SP algorithm, the ESA algorithm makes use of greener paths, which also results in longer paths, as shown in Figure 2. A more frequent re-routing decision also tends to increase the hop count number. At the same time, pure ESA results in a higher connection blocking probability than the SP algorithm, as shown in Figure 3. Similar to the pure load balancing algorithm, the routing decision using the ESA algorithm depends on the update of energy sources information in routing tables. ESA routing decisions tend to select greener path, which would create certain "hot pot" effect on the greener paths. The "hot pot" effect gets worse when the routing table information remains unchanged for a longer period of time, which explains the growing blocking probability with the increase of EnergyChangeInterval for both the ESA algorithm and the pure load balancing algorithm. On the other hand, the blocking probability is higher when shorter EnergyChangeInterval is used when applying re-routing optimization, with the most saving optimization (ESA + reroute 30\%) resulting in the highest blocking. This is because the large number of re-routing attempts (occupying resources whether successful or unsuccessful during the set up time) can decrease the chance of setting up new connection requests successfully. As shown in Figure 3, the load balancing algorithm performs better than the others in terms of blocking probability with shorter update intervals, which gives a good indication of ways to lower blocking probability for selected optimization method. By applying the combining algorithm (ESA + reroute $30 \%+$ load balancing 50\%), a lower blocking probability can be obtained together with a lower hop count for longer EnergyChangeInterval values, which comes at the expense of a relatively small increase in $\mathrm{CO}_{2}$ emissions. 


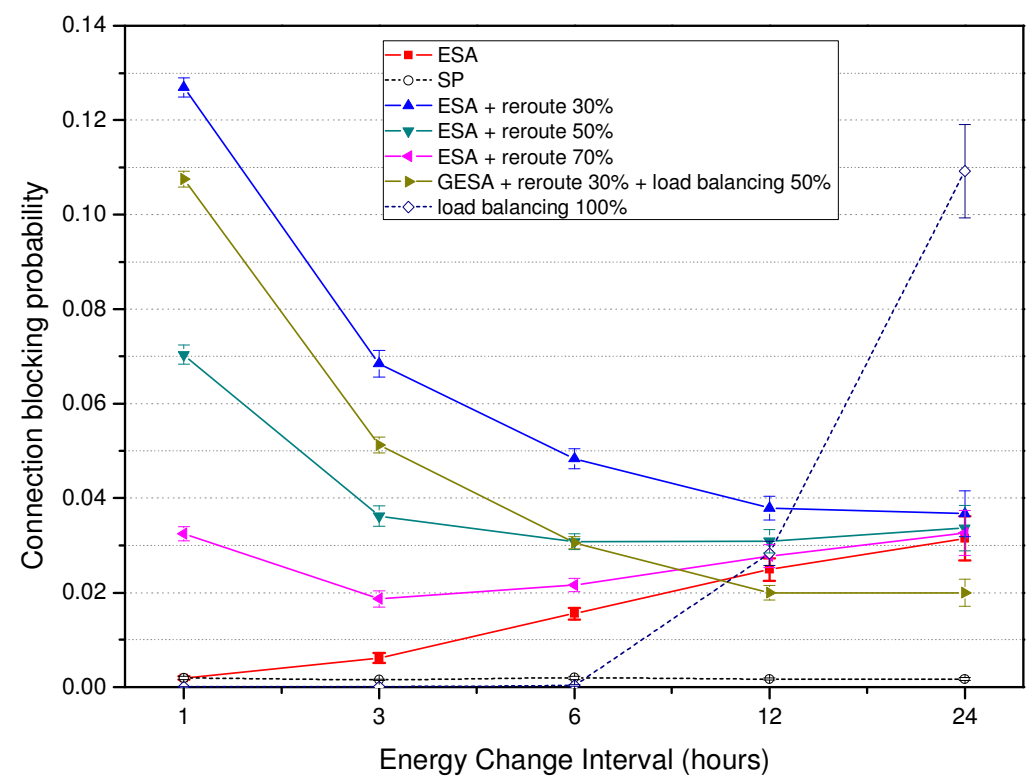

Figure 3. Average connection blocking probability.

\section{CONCLUSIONS}

In this paper, the authors proposed the ESA routing and re-optimization algorithms to reduce $\mathrm{CO}_{2}$ emissions in dynamic GMPLS controlled core optical networks. Results show that ESA routing algorithm can decrease $\mathrm{CO}_{2}$ emissions, compared to traditional SP and pure load balancing algorithms, under higher EnergyChangeInterval. Employing re-routing strategies to ESA algorithm can further bring down $\mathrm{CO}_{2}$ emissions at the expense of increased blocking probability. By adding load balancing criteria, the algorithm can reach the lowest blocking probability in certain range. A clear trade-off is observed between connection blocking probability and obtained $\mathrm{CO}_{2}$ savings, which must be addressed accordingly. However, any optimization method proposed would cause extra signalling messages flooded over the entire network area, which adds cost and complexity.

\section{ACKNOWLEDGEMENTS}

This work was supported in part by the COST Action IC0804 on Energy Efficiency in Large Scale Distributed Systems, the Spanish Ministry of Science and Innovation under the DOMINO project (TEC2010-18522), the Catalan Government under the contract SGR 1140 and the IUE/ESF under the grant FI-201000740. The authors also acknowledge the support received from OPNET Technologies Inc.

\section{REFERENCES}

[1] BONE project, WP 21 Topical Project Green Optical Networks: Report on year 1 and updated plan for activities, NoE, FP7-ICT-2007- 216863, Dec. 2009.

[2] X. Dong et al.: "IP over WDM Networks Employing Renewable Energy Sources", in Journal of Lightwave Technology, vol. 29, Jan., 2011.

[3] M. Xia et al.: "Green Provisioning for Optical WDM Networks", in Journal of Selected Topics in Quantum Electronics, vol. 17, Mar., 2011.

[4] S. Ricciardi et al.: "Energy-Aware RWA for WDM Networks with Dual Power Sources", IEEE International Conference on Communications (ICC 2011), Jun. 5-9, 2011

[5] S. W. Lee et al.: "A Distributed Link Management Algorithm for Energy Efficient IP Networks," IEEE Globecom 2011, Dec., 2011.

[6] L. Liu et al.: "Rightsizing Bundle Link Capacities for Energy Savings in the Core Network," IEEE Globecom 2011, Dec., 2011.

[7] E. Mannie: "Generalized Multi-Protocol Label Switching (GMPLS) Architecture," RFC 3945, Oct., 2004.

[8] D. Katz et al.: "Traffic Engineering (TE) Extensions to OSPF Version 2," RFC 2370, Sep., 2003.

[9] R. Aggarwal et al.: "Extensions to Resource Reservation Protocol - Traffic Engineering (RSVP-TE) for Point-to-Multipoint TE Label Switched Paths (LSPs)," RFC 4875, May, 2007.

[10] OPNET Technologies, Inc., http://www.opnet.com.

[11] J. Wang et al.: "Green-Aware Routing in GMPLS Networks", ICNC 2012 workshop, Maui, Jan. 2012. 